\title{
LA MÉTAPHORE GUERRIÈRE DANS LA COMMUNICATION POLITIQUE
}

\author{
Gilles Gauthier $^{1}$
}

[Le conflit physique] apparaît partout dans le règne animal et nulle part autant que dans la race humaine. Les animaux se battent pour obtenir ce dont ils ont besoin (la nourriture, la sexualité, le territoire, la domination), car d'autres animaux désirent les mêmes choses et veulent les empêcher de les obtenir. Il en est de même pour les humains, à cette différence près que nous avons développé des techniques beaucoup plus élaborées pour parvenir à nos fins. Comme nous sommes des "animaux rationnels", nous avons institutionnalisé nos conflits de diverses manieres, parmi lesquelles la guerre.

(...) un des avantages qu'il y a à être un animal rationnel est qu'on peut obtenir ce qu'on veut sans courir le risque d'un conflit physique réel. C'est à cette fin que nous autres hommes avons inventé l'institution sociale de la controverse. Nous discutons sans cesse pour obtenir ce que nous voulons et parfois seulement ces discussions "dégénèrent" en violences physiques. Ces batailles verbales, nous les vivons à peu près sur le même mode que les batailles physiques ${ }^{2}$.

1 Professeur au Département d'information et de communication de l'Université Laval. Une version préliminaire et partielle de ce texte a été présentée au XVe congrès international des linguistes tenu à Laval en août 1992.

2 George LaKoFF.

Recherches en communication, $\mathrm{n}^{\circ} 1$ (1994). 
Si, à la façon de Foucault, l'on cherchait à identifier les principaux constituants de l'épistèmè de la recherche contemporaine en sciences humaines et sociales, il y a de fortes chances que la métaphore serait retenue. La métaphore n'est plus, depuis une vingtaine d'années, une simple "figure de rhétorique": elle est devenue le point d'ancrage à partir duquel grand nombre de linguistes, sémioticiens, sémiologues, sociologues, ethnologues, philosophes et autres déterminent et développent leur objet d'étude ${ }^{1}$.

La science politique n'échappe pas à ce courant. Un assez grand nombre de travaux traitent de quelque aspect de la relation entre métaphore et politique. Quelques-unes de ces recherches tendent à faire valoir le rôle de la métaphore dans la construction de la réalité politique (voir, par exemple, Minogue ${ }^{2}$ ou son importance dans la constitution même de la connaissance politique, par exemple, Miller ${ }^{3}$ ). Certaines (comme Epps ${ }^{4}$ et Vernon ${ }^{5}$ ) vont même jusqu'à soutenir que l'activité ou l'institution politique est elle-même un ensemble métaphorique. Toutefois, la majorité des études cherchent, sur un plan moins spéculatif et plus analytique, à examiner l'utilisation de la métaphore dans la communication politique ${ }^{6}$. Si certaines portent sur la métaphore dans le traitement journalistique et massmédiatique de

1 Pour se convaincre de l'étendue de l'intérêt qui est aujourd'hui accordé à la métaphore dans l'ensemble de la recherche scientifique, il suffit de considérer les volumineuses bibliographies qui lui sont consacrées. Voir, par exemple, Jean-Pierre VAN NOPPEN et Édith HOLs, Metaphor II. A Classified Bibliography of Publications 1985 to 1990, Amsterdam/Philadelphia, John Benjamins Publ. Co., 1990; JeanPierre VAN NOPPEM et al., Metaphor. A Bibliography of Post-1970 Publications, Amsterdam/Philadelphia, John Benjamins Publ. Co., 1985 et SHIBLEs et A. WhARREN, Metaphor. An Annoted Bibliography and History, Whitewater, Wisconsin, The Language Press, 1971.

2 Minogue et KenNeth, "The Place of Metaphor in the Construction of Political Reality", in Maurice Cranston et Peter MAIR (eds), Langage et Politique. Language and Politics, Bruxelles, Buyaut, 1982, p. 123-135.

3 E. F. MILlER, "Metaphor and Political Knowledge", in American Political Science Review, 73, 1979, p. 155-170.

4 EPPS et GARreTT, "Politics as Metaphor", in Virginia Quarterly Review, 55, 1979, p. $75-98$.

5 Richard VERnON, "Politics as Metaphor: Cardinal Newman and Professor Kuhn", in Review of Politics, 41, 1979, p. 513-535.

${ }^{6}$ Ici encore, les bibliographies sont indicatrices de l'importance de ce champ de recherche. Voir, entre autres, Dennis JACKSON, E.A. NICKERSON et F.B. JACKSON, "The Style of Political Discourse: Annoted Bibliography II: Metaphor and Semiotics", in Style, 16, 1982, p. 143-148, et J. SEAmaN, "The Style of Political Discourse: An Annoted Bibliography", in Style, 8 (3), 1974, p. 477-528. 
l'actualité politique (par exemple Mumby et Spitzack ${ }^{1}$, Larson ${ }^{2}$ et Cierreux ${ }^{3}$, la plus grande partie des travaux de ce type tentent d'éclairer l'utilisation qui est faite de la métaphore dans le discours politique lui-même. Des recherches envisagent la question d'un point de vue très large: relativement au langage général de la politique (voir, par exemple, Kauffman ${ }^{4}$, Paine ${ }^{5}$, Powell ${ }^{6}$ et Zashin et Chapman ${ }^{7}$, au discours politique contemporain (Howe ${ }^{8}$ ou au langage plus spécifiquement présidentiel, Kitchen ${ }^{9}$ ). D'autres travaux constituent des "analyses de cas" en ce qu'ils cherchent à analyser la fonction de la métaphore dans le discours d'hommes et de femmes politiques ou encore dans certains discours idéologiques bien identifiés. C'est ainsi que des études ont été effectuées sur la rhétorique d'Hitler, Roosevelt, Nixon, Reagan, Cuomo et bien d'autres figures politiques (voir, parmi d'autres, Henry ${ }^{10}$, Etienvre ${ }^{11}$, Hankins ${ }^{12}$, Perry ${ }^{13}$, Johnson ${ }^{14}$, Ryan ${ }^{15}$,

1 D.K. MUMBY et C. SPITZACK, "Ideology and Television News: A Metaphoric Analysis of Political Stories", in Central States Speech Journal, 34, 1983, p. 162 171.

2 C.U. LARSON, "Media Metaphors: Two Models for Rhetorically Criticizing the Political Television Spot Advertisement", in Central States Speech Journal, 33 (4), 1982, p. 533-546.

3 B. Cierreux, "Le sport: une mine d'or pour la presse politique", in Le Ligueur, 46, 1981, p. 4.

4 Charles Kauffman, "Names and Weapons", in Communication Monographs, 56, 1989, p. 273-385.

5 R. PAINE, "The Political Uses of Metaphor and Metonym: An Exploratory Statement", in Politically Speaking: Cross-Cultural Studies of Rhetoric, Philadelphia, Institute for the Study of Human Issues, 1981, p. 87-200.

6 J. Enoch Powell, "The Language of Politics, in L. MichAELS et C. Ricks (eds), The State of the Language, Berkeley, University of California Press, 1980, p. 432439.

7 E. Zashin et P.C. ChAPMAN, "The Uses of Metaphor and Analogy Toward a Renewal of Political Language", in Journal of Politics, 36 (2), 1974, p. 290-326.

8 Nicholas Howe, "Metaphor in Contemporary American Political Discourse", in Metaphor and Symbolic Activity, 3 (2), 1988, p. 87-104.

9 W. KITCHEN, "Imagery, Emotion, and Cause and Effect in Presidential Language", in International Political Science Review, 8, 1987, p. 111-119.

10 D. HENRY, "The Rhetorical Dynamic of Mario Cuomo's 1984 Keynote Address: Sithor", in Southem Speech Communication Joumal, 53, 1988, p. 105-120.

11 J.-P. ETtEnVRE, "Du jeu comme métaphore politique. Sur quelques textes de propagande royale diffusés en Espagne à l'avènement des Bourbons", in Poétique, 56,1983 , p. 397-415.

12 S.R. HANKIns, "Archetypal Alloy: Reagan's Rhetorical Image", in Central States Speech Journal, 343, 1983, p. 33-43.

13 PERRY, "Rhetorical Functions of the Infestation Metaphor in Hitler's Rhetoric", in Central States Speech Journal, 34, 1983, p. 229-235. 
Howe' et Clark ${ }^{2}$. D'autres études plus thématiques, portent sur le discours marxiste (Ganssman ${ }^{3}$ et Angenot et Suvin ${ }^{4}$ ), la guerre froide $\left(\right.$ Medhurst $^{5}$, Ivie $^{6}$ ) ou encore des causes plus "locales" comme celle des réfugiés palestiniens (Johnson ${ }^{7}$ ). D'autres travaux, par exemple Bosman $^{8}$, portent sur les effets persuasifs des métaphores politiques.

Je m'intéresserai ici à une métaphore ou plutôt à ce qui m'apparaît être l'un des principaux réseaux métaphoriques du discours politique: la métaphore guerrière. La politique, par ceux là même qui en sont les artisans et aussi par ceux qui en sont les observateurs et analystes, est souvent dite être une "arêne", un "combat", une "lutte", une "bataille" mettant aux prises des "opposants", "adversaires" et "ennemis", faisant l'objet d'une "victoire" ou d'une "défaite" au cours de "campagnes", "débats" et autres formes d" "affrontements" suivant des "manœuvres", "stratégies", "tactiques" et autres considérations polémologiques.

La métaphore guerrière est manifestement un constituant et une illustration de la communication politique. Je ne chercherai pas ici à en rendre compte dans toutes ses dimensions. Je me limiterai à traiter $\mathrm{du}$ problème plus circonscrit mais très certainement fondamental du statut sémantique de la métaphore guerrière dans le discours politique.

14 J.P. JOHNSON, "Nixon's Use of Metaphor: The Real Nixon Tapes", in Psychological Review, 66 (2), 1979, p. 263-274.

15 H.R. RYAN, "Roosevelt's First Inaugural: A Study of Technique", in Quarterly Journal of Speech, 65 (2), 1979, p. 137-149.

1 James Howe, "Carrying the Village: Cuna Political Metaphors", in David SAPIR et Christopher J. Crocker (eds), The Social Use of Metaphor, Pennsylvania, University of Pennsylvania Press, 1977, p. 132-163.

2 Thomas ClARK, "Rhetorical Image-Making: A Case Study of the Thomas Paine William Smith Propaganda Debates", in Southern Speech Communication Journal, 40,1975 , p. 248-261.

3 H. GANSSMAN, "Abstract Labor as a Metaphor: a Comment", in History of Political Economy, 20 (3), 1988, p. 461-470.

4 M. ANGENOT et D. Suvin, "L'implicite du manifeste: Métaphores et imagerie de la démystification dans le Manifeste Communiste", in Études Françaises, 16 (3-4), 1980, p. 43-67.

5 Martin Medhurst, Cold War Rhetoric: Strategy, Metaphor and Ideology, Westport. Conn., Greenwood Press, 1990.

6 R.L. IVIE, "Metaphor and the Rhetorical Invention of Cold War Idealists", in Communication Monographs, 54, 1987, p. 165-182 et Idem, "Literalising the Metaphor of Soviet Savagery: President Truman's Plain Style", in Southern Speech Communication Journal, 51, 1986, p. 91-105.

7 N. JoHnson, "Palestinian Refugee Ideology: An Enquiry into Key Metaphors", in Journal of Anthropological Research, 34 (4), 1978, p. 524-539.

8 J. Bosman, "Persuasion Effects of Political Metaphors", in Metaphor and Symbolic Activity, 2, 1987, p. 97-113. 
Je procéderai en prenant appui sur la (sous-)théorie de la métaphore mise en avant par John Searle dans le cadre de sa théorie des actes de langages. Après avoir assez rapidement décrit et caractérisé la conception searlienne de la métaphore, j'examinerai la question de la littéralité de la métaphore guerrière dans la communication politique, d'abord en me mettant à la recherche de sa signification littérale, ensuite en concluant à sa nature littérale paradoxale.

\section{Métaphore et actes de langage: la théorie de Searle}

Depuis maintenant plus de vingt ans, le philosophe américain

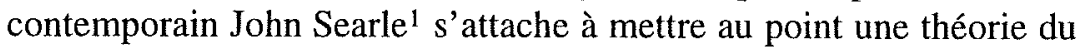
langage en vertu de laquelle il ne consisterait pas, comme on l'a traditionnellement pensé, essentiellement en une description du monde mais plutôt en l'accomplissement de différentes actions. Searle se trouve ainsi au point de passage d'une conception sémantique (au sens positiviste "dur" du terme) à une conception pragmatique du langage.

Dans sa théorie des actes de langage (speech acts), il cherche à démontrer que dans sa constitution et sa structuration mêmes, le langage est fondamentalement marqué par l'usage qu'en font ses utilisateurs. Selon Searle, parler, c'est toujours accomplir un certain acte (asserter, promettre, ordonner, etc.). Cet acte de langage est constitué de deux composants abstraits: une force illocutoire, l'action en laquelle il consiste -par exemple, une promesse et non pas un ordre-; et un contenu propositionnel, la teneur de cet acte-par exemple, la promesse de faire telle chose et non pas telle autre chose-. La forme ou le modèle (général) des actes de langage est $F(p)$ : $F$ représentant leur force illocutoire et (p) leur contenu propositionnel. Toujours d'après Searle, cette structuration des actes de langage se reflète dans les énoncés qui servent à les accomplir; un énoncé serait ainsi muni d'un indicateur de force illocutoire (qui peut être un performatif comme "je promets" ou, plus simplement, le mode et le temps du verbe) et un indicateur de contenu propositionnel (les mots et expressions de l'énoncé).

1 Voir surtout John R. SEARLE, Speech Acts. An Essay in the Philosophy of Language, Cambridge, Cambridge University Press, 1969. Idem, Expression and Meaning. Studies in the Theory of Speech Acts, Cambridge, Cambridge University Press, 1979. Idem, Intentiality. An Essay in the Philosophy of Mind, Cambridge, Cambridge University Press, 1983. 
L'une des grandes et nouvelles idées de Searle est que la force illocutoire contribue à la signification d'un énoncé. Searle soutient ainsi que le sens d'un énoncé n'est pas donné par sa seule teneur propositionnelle mais aussi par l'acte que son emploi équivaut à accomplir. Soit l'énoncé "Je promets d'être là". D'après Searle, sa signification et par extension sa compréhension seraient déterminées non seulement par son contenu de représentation: l'état de choses stipulant ma présence, mais également par la force illocutoire de promesse: l'acte de promettre accompli par l'utilisation de l'énoncé. Cette idée peut, je crois, être considérée comme la thèse searlienne globale sur la signification. Elle pose ou elle affirme sans vraiment le démontrer que l'acte de langage que sert à accomplir un énoncé contribue à sa signification. Cette thèse est en quelque sorte programmatique; elle ouvre une voie de recherche. Elle indique aussi que Searle tente de passer d'une sémantique interne au langage à une sémantique (c'en est encore une: c'est toujours de signification dont il s'agit) moins fermée qui tient également compte de l'usage effectif du langage.

Cette thèse, Searle cherche à l'expliciter dans ce qui peut être considéré comme sa théorie unifiée de la signification. La principale caractéristique de cette théorie est qu'elle prend en consédération la grande capacité expressive du langage; elle porte non seulement sur l'énonciation littérale mais également sur les diverses formes nonlittérales d'énonciation. Searle aborde ainsi tout autant le discours disons "standard" (direct et littéral). Ce que j'appelle la théorie searlienne unifiée de la signification est en fait constituée d'un regroupement de sous-théories: une sous-théorie de la signification littérale et une série de sous-théories de signification non-littérale.

Toutes ces théories relèvent d'une vue d'ensemble commune. C'est précisément en cela qu'elles peuvent être dites constituées une théorie unifiée. La question générale de la signification est traitée par Searle à partir d'une distinction fondamentale entre deux concepts de signification: la signification propre de l'énoncé ou de la phrase (sentence meaning) et la signification de son emploi par le locuteur -la signification, en quelque sorte de l'énonciation effective de l'énoncé- (utterance or speaker's meaning). La signification d'un énoncé, le sens que lui reconnaissent tous ses usagers, n'est pas identique en nature à la signification projetée par un locuteur quand il y a recours, ce qu'on nomme souvent son "vouloir dire". Une fois cette distinction reconnue, deux possibilités se présentent. Soit que la signification de l'énonciation coïncide à la signification de l'énoncé, 
c'est-à-dire que le locuteur veuille dire tout à fait ce que signifie l'énoncé, auquel cas nous nous trouvons devant un cas d'énonciation littérale. Soit que la signification de l'énonciation se démarque de quelque façon de la signification de l'énoncé, quand en utilisant tel énoncé un locuteur veut en fait signifier autre chose que la signification de cet énoncé; nous avons alors affaire à un cas complexe d'énonciation non-littérale. Signalons, par ailleurs, que Searle soutient qu'il est toujours possible à un locuteur de signifier exactement ce qu'il veut signifier, c'est-à-dire de trouver un énoncé dont la signification corresponde parfaitement à la signification de son énonciation. C'est ce qu'il nomme le "principe d'exprimabilité" (principle of expressibility). Selon Searle, par conséquent, tous les cas complexes d'énonciation non-littérale sont réductibles à un cas simple d'énonciation littérale.

La sous-théorie de la signification littérale de Searle ${ }^{1}$ porte le statut sémantique de l'énoncé et a trait, par extension, au cas simple d'énonciation: quand la signification de l'énonciation correspond en tout point à la signification de l'énoncé, quand le locuteur veut dire intégralement ce que l'énoncé qu'il profère veut dire. On pourrait penser que l'admission par Searle d'une signification propre de l'énoncé sape immédiatement sa thèse sur la signification, à savoir, encore une fois, que la force illocutoire ou, plus généralement l'acte de langage qu'il permet d'accomplir, contribue à la signification de l'énoncé. L'énoncé n'a-t-il pas, dès lors, une signification pour ainsi dire antérieure à son emploi effectif et donc indépendante de cette utilisation? Sa reconnaissance de la signification de l'énoncé ne conduit pas Searle à cette contradiction. Il soutient, en effet, dans sa sousthéorie de la signification littérale, que la signification de l'énoncé n'est pas contextuellement indépendante.

Selon lui, la signification littérale d'un énoncé dépend essentiellement d'un ordre contextuel extra-langagier. Plus précisément, il soutient qu'un énoncé n'a de signification que par rapport à un ensemble de prémisses d'arrière-plan (background assumptions) relatives au contexte dans lequel l'énoncé peut être utilisé de manière appropriée pour accomplir un acte de langage. Autrement dit, ce serait eu égard à l'acte de langage que son emploi sert à accomplir qu'un énoncé serait même muni d'une signification littérale. Celle-ci serait ainsi toute relative: la signification d'un énoncé ne lui serait pas

1 John R. SEARLE, "Literal Meaning", in Erkenntnis, 13 (1), 1978, p. 207-224. 
intrinsèque mais dépendrait de certains éléments de la vision du monde du locuteur. On pourrait même envisager la possibilité qu'un énoncé ait diverses significations littérales, chacunes relatives à un ensemble différent de prémisses d'arrière-plan. On pourrait aussi, à la suite de la théorie searlienne de la signification littérale, formuler l'hypothèse historique qu'un grand nombre (la majorité, la totalité?) des énoncés des langues naturelles ont eu, à travers les âges, plusieurs significations littérales différentes du fait de changements dans les prémisses d'arrière-plan en déterminant le sens.

A la lumière de cette théorie de la signification littérale et de la distinction entre signification de l'énoncé et signification de l'énonciation, la thèse de Searle sur la signification se précise grandement. Sa formulation stricte devient la suivante: la force illocutoire d'un acte de langage contribue à la signification de l'énoncé utilisé (littéralement et dans un contexte approprié) pour accomplir cet acte de langage de cette force illocutoire. Autrement dit, pour déterminer quelle force illocutoire ou quel acte de langage participe à la signification d'un énoncé, il faut considérer strictement le cas d'énonciation littérale de cet acte.

Dans un cas d'énonciation littérale, un locuteur signifie exactement ce que l'énoncé qu'il profère signifie relativement à un ensemble assez bien déterminé de prémisses d'arrière-plan. Laissons là la question de cette signification littérale pour aborder celle de l'énonciation non-littérale. Les sous-théories locales de Searle sur la non-littéralité portent sur quelques-une des façons suivant lesquelles ce qu'un locuteur signifie s'écarte de ce que signifie l'énoncé qu'il emploie. L'une de ces théories porte sur la métaphore (Searle a également élaboré une sous-théorie de l'acte de langage indirect et esquissé une sous-théorie de l'expression ironique).

Selon Searle', le cas le plus général et le plus simple d'énonciation métaphorique est celui où un locuteur veut signifier un état de choses "S est R" en employant un énoncé plutôt de la forme "S est P". La grande caractéristique de la métaphore est que son sens est complètement différent de celui du propos du locuteur: la signification de l'énonciation se détache radicalement de la signification de l'énoncé. Quand il utilise une métaphore, un locuteur ne veut absolument pas dire "S est P" mais quelque chose de tout à fait autre, "S est R". Fondamentalement, la métaphore se fonde sur une certaine

1 John R. SEARLE, Expression..., op. cit., p. 76-116. 
correspondance entre "P" et "R". Elle fonctionne en vertu de quelque rapprochement ou parenté qui peut être établi entre "P" et " $R$ "; en fonction donc d'une propriété ou d'un trait commun à "P" et "R". Selon Searle, ce rapport de similitude est lâche et multiple. Il peut être identifié par un ensemble indéfini de principes -lui-même repère sept principes distincts ${ }^{1}$.

La principale préoccupation de Searle au sujet de la métaphore est de savoir comment peut s'opérer le transfert de sens de "S est P" à "S est R"; Searle, autrement dit, cherche à déterminer comment le locuteur peut parvenir à signifier "S est R" en disant "S est P", "P" étant, répétons-le, radicalement différent de "R". En fait, Searle traite de ce problème du point de vue de l'auditeur; la question devient alors de savoir comment un auditeur peut parvenir à comprendre que le locuteur signifie "S est R" alors que ce dernier dit quelque chose de tout à fait différent, "S est P". Searle prétend être en mesure de démonter le processus d'inférence suivi par l'auditeur au moyen d'un appareillage théorique comprenant la théorie des actes de langage, des principes d'ordre conversationnels et l'information factuelle dont dispose les interlocuteurs. Cet appareillage lui permet en fait de rendre compte de tous les cas d'énonciation non-littérale qu'il analyse.

Dans le cas de la métaphore, la procédure qu'il permet de mettre au jour comporte trois phases: de détection, de recension et de restriction. En présentant les choses simplement -c'est-à-dire en escamotant une discussion des principes conversationnels et de l'information requise de la part des interlocuteurs- ces trois étapes peuvent être caractérisées de la façon suivante.

- Le locuteur doit d'abord détecter une défectuosité dans l'énonciation de "S est P"; il doit en quelque sorte rejeter la possibilité que le locuteur cherche vraiment à signifier "S est P" en disant "S est P" et, par conséquent, se mettre à la recherche d'une signification autre que "S est P".

- Pour ce faire, l'auditeur doit ensuite recenser et supputer les différents aspects de "P" en fonction desquels le locuteur pourrait devoir dire "S est comme P" (et non pas littéralement "S est P" en disant néanmoins " $S$ est $P$ ").

- Finalement, l'auditeur doit restreindre ces traits de "P" à ceux qui peuvent être effectivement prédiqués de "S", compte tenu de

1 lbid., p. 107-110. 
l'identité et des propriétés de ce "S" et ne retenir qu'une seule ou quelques possibilités pertinentes.

Sommairement, la procédure proposée par Searle peut être décrite comme suit: après avoir déterminé que la signification de l'énonciation n'est pas littérale et est donc distincte de la signification de l'énoncé " $S$ est $P$ ", l'auditeur doit identifier un ou des énoncés "S est R" dont la signification littérale serait identique à la signification de l'énonciation.

\section{Le problème de la signification littérale des métaphores guerrières du discours politique}

Le discours politique est truffé d'expressions métaphoriques de toutes sortes. Il comporte, par exemple, des métaphores corporelles ("le cœur du pouvoir", "la tête du parti", "le bras droit du chef") et des métaphores naturelles ("un vent de changement", "une vague conservatrice"). Un certain nombre de métaphores politiques sont relatives à la nature conflictuelle de l'activité politique. Ces métaphores sportives ("l'équipe libérale", "marquer des points"), militaires ("les troupes partisannes", "un château fort socialiste") ou plus largement guerrières ("se porter à l'attaque", "triompher de l'adversaire") peuvent sûrement faire l'objet de sous-catégorisations. Un certain nombre de travaux leur ont été consacrés ${ }^{1}$. Il existe même au moins une analyse des métaphores guerrières de la communication politique ${ }^{2}$ qui prétend tenir compte de la théorie des actes de langage de Searle.

Sans égard pour ces différenciations, mon intérêt porte ici sur l'ensemble des métaphores de la communication politique qui ont trait à son cadre oppositionnel. Ce que donc, pour les fins de mon examen, j'appelle une métaphore "guerrière", c'est une métaphore par laquelle est exprimé quelque trait de l'affrontement politique. Mon analyse est restreinte aux seules métaphores simples: celles qui qualifient un individu ou qui spécifient quelque trait de son comportement. Le type de métaphores examinées par Searle, celles de la forme "S est P"

1 Voir, entre autres, Nicholas Howe, op. cit., Kenneth Minogue, op. cit., Bernard P. KIERMAN, "War and Zero-Sum Games", in Virginia Quarterly Review, 53, 1977, p. 17-31 et Ike BaLbus, "Politics as Sports: the Political Ascendancy of the Sports Metaphor in America", in Montly Review, 26 (mar.), 1975, p. 26-39.

2 F.R. DAllmayr, Language and Politics - Why Does Language Matter to Political Philosophy, Notre-Dame, University of Notre-Dame Press, 1983. 
appartiennent à cette catégorie générale. Voici quelques exemples de métaphores simples de la communication politique: "Tel parti est en pleine déroute", "X a mis sa tête sur le billot", " $X$ préconise une trêve constitutionnelle", "X s'élève contre tel empiètement", "X a brûlé ses dernières cartouches", " $X$ pratique la politique de la terre brûlée", " $X$ a mis le point sur la table", "X torpille les efforts de ses ministres", "X se retrouve le couteau sur la gorge".

Dans les termes de la sous-théorie de la signification non-littérale de la métaphore de Searle, ces métaphores guerrières de la communication politique peuvent être traitées de la façon suivante. Soit, par exemple, l'énonciation de "X est belliqueux". L'auditeur y détecte une défectuosité qui l' amène à supposer que le locuteur ne veut pas vraiment signifier que " $X$ est belliqueux" ("S est P") et qu'il doit donc rechercher une autre signification ("S est R"). Autrement dit, l'auditeur convient que la signification de l'énonciation de " $\mathrm{X}$ est belliqueux", ce que le locuteur veut vraiment dire, se démarque de la signification de l'énoncé et il doit par conséquent se mettre en quête d'un autre énoncé dont la signification serait identique à la signification de l'énonciation de "X est belliqueux". En vertu du principe d'exprimabilité, cet énoncé doit exister (ou peut être créé); de même, le locuteur aurait pu y avoir recours pour s'exprimer littéralement: s'il a choisi d'énoncer "S est P" pour signifier "S est R", il avait néanmoins la possibilité d'énoncer "directement" c'est-à-dire littéralement "S est R".

Deux points ici sont fondamentaux: la reconnaissance de la métaphore et la nécessité conséquente de trouver une signification littérale à l'énonciation. D'abord, l'emploi (commun, habituel) de "X est belliqueux" dans le discours politique est certainement métaphorique. Le terme "belliqueux" y est manifestement utilisé dans le sens figuré que lui reconnaît le dictionnaire: pour désigner quelqu'un "qui aime, qui cherche le combat, la dispute". La reconnaissance du caractère inadéquat de l'emploi du terme (dans son interprétation stricte) et donc de l'écart caractéristique de la métaphore entre un "S est $\mathrm{P}$ " proféré et un "S est P" visé se fonde sur une inadéquation entre le sens littéral de "X est belliqueux" et le contexte d'énonciation. Il apparaît inadéquat d'utiliser cet énoncé, dans son sens littéral, relativement à un état de choses qui n'est pas marqué par la guerre: il ne fait pas sens, à strictement parler, de qualifier un individu de "belliqueux" dans un contexte autre que celui d'état de guerre. Dans un discours parfaitement littéral (où tout "S est P" serait employé pour signifier 
effectivement "S est P"), le terme "belliqueux" et le contexte de communication politique seraient incompatibles (sauf dans le cas, évidemment, d'un réel affrontement armé entre deux forces politiques).

Si donc la signification de l'énonciation politique n'équivaut pas à la signification de l'énoncé " $X$ est belliqueux", quelle est-elle? Le rejet de "S est P" entraîne la recherche d'un "S est R". Cette quête de la signification véritable de l'énonciation revient à l'identification d'un autre énoncé dont la signification littérale serait, elle, identique à la signification de l'énonciation. Il s'agirait là de l'aspect central de la métaphore -telle du moins que Searle la caractérise-: à une énonciation non-littérale dont la signification n'est pas explicitement donnée par l'énoncé utilisé doit correspondre, suivant le principe d'exprimabilité, une énonciation littérale, c'est-à-dire que doit donc exister un énoncé qui, lui, en vertu de sa signification littérale livre la signification littérale de l'énonciation et dont l'emploi aurait résulté en une énonciation littérale. Le recours métaphorique de l'énoncé " $X$ est belliqueux", dans un contexte de communication politique, implique ainsi l'existence d'un autre énoncé qui porterait la signification de l'énonciation.

Quel peut être cet énoncé? Quel serait le "S est R" du "S est P" que constitue "X est belliqueux"? Qu'est-ce qu'un locuteur peut bien vouloir signifier vraiment quand il dit " $\mathrm{X}$ est belliqueux"? Telles sont les questions essentielles qui se posent au sujet des métaphores guerrières de la communication politique. La thèse que je soutiens, c'est qu'il n'y a pas de "S est R" à trouver à ces métaphores; qu'il n'existe pas d'énoncé dont la signification littérale correspondrait à la signification de l'énonciation de l'usage métaphorique de " $\mathrm{X}$ est belliqueux".

Le problème étonnant et crucial que présentent les métaphores guerrières de la communication politique est qu'il n'apparaît pas possible de leur trouver un sens littéral, plus précisément de trouver un énoncé qui expliciterait, en vertu de sa signification littérale, la signification de l'énonciation. Eu égard à la procédure de mise au point par Searle, la difficulté n'est pas à la troisième étape, d'isoler et de retenir une signification parmi différentes possibilités mais, à la seconde étape, d'établir même ces différentes possibilités. Autrement dit, le problème de la métaphore guerrière de la communication politique réside dans l'inexistence de candidats au titre d'énoncé littéral, de "S est R". 


\section{La métaphore guerrière de la communication politique: une métaphore littérale}

Ce constat ne permet d'ouvrir qu'une seule porte de sortie: celle qui consiste à reconnaître ou à supposer que les métaphores guerrières de la communication politique -en tout cas certaines d'entre ellessont en fait employées littéralement. Quand pour reprendre le même exemple que plus haut, un locuteur dit "X est belliqueux", il veut vraiment dire ce que l'énoncé signifie: quelque chose comme $X$ recherche la controverse, l'affrontement. De ce point de vue, la signification de l'énonciation rejoint tout à fait la signification de l'énoncé et il n'y a pas d'écart entre "S est R" et "S est P", "R" et "P" étant en fait (à toutes fins utiles) identiques.

Mais alors, la métaphore guerrière de la communication politique constitue un très grand paradoxe: elle est une métaphore littérale. Un énoncé comme "X est belliqueux" est bel et bien métaphorique, c'està-dire que son emploi donne lieu à une forme d'énonciation non-littérale... qui, par ailleurs, s'avère être bilatérale! Comment comprendre ce phénomène? Reprenons l'un après l'autre les principaux points de l'analyse. "X est belliqueux" est une réelle métaphore parce que son utilisation, à strictement parler, est manifestement inapproprié au contexte de la communication politique. La preuve en est que l'auditeur détecte une défectuosité et se met à la recherche d'une interprétation autre: d'un "S est R" que pourrait vouloir signifier le locuteur en disant "S est P". Mais ne trouvant pas de candidat valable au titre de "S est R", l'auditeur doit finalement revenir se rabattre sur "S est P" ou plutôt doit se rendre à l'évidence que le "S est R" que veut signifier le locuteur est précisément malgré tout le "S est $\mathrm{P}$ " qu'il profère. Ce déplacement semble s'opérer, globalement, de la façon suivante: par rapport à la guerre (la vraie guerre), "X est belliqueux" est une métaphore et son énonciation est donc non-littérale; mais du point de vue de la communication politique (d'une certaine conception "guerrière" de la communication politique), l'emploi de l'énoncé devient cependant tout à fait littéral et, pour ainsi dire, il perd de la sorte son caractère métaphorique.

Dans la sous-théorie searlienne de la métaphore, ce processus correspond à la métaphore morte (dead metaphor). Dans les termes de la théorie de Searle, une métaphore est une métaphore morte quand la signification littérale originale d'un énoncé est abolie au profit de la signification non-littérale de son énonciation, quand donc cette signi- 
fication non-littérale antérieure devient la nouvelle signification littérale de l'énoncé qui perd, pour ainsi dire, du point de vue de l'énonciation, son statut métaphorique. C'est tout à fait ce qui arrive à notre énoncé " $X$ est belliqueux": sa signification littérale originale (quelque chose comme " $\mathrm{X}$ aime le combat, la dispute de la véritable guerre") est remplacée par la signification métaphorique non-littérale de son énonciation (quelque chose comme " $\mathrm{X}$ aime le combat, la dispute de la politique") qui devient ainsi la nouvelle signification littérale de l'énoncé.

\section{Conclusion:}

\section{la communication politique, une communication stratégique}

Le paradoxe de la métaphore guerrière de la communication politique (encore une fois, cette transformation de l'emploi d'un énoncé comme " $X$ est belliqueux" d'une énonciation non-littérale à une énonciation littérale) s'explique fondamentalement par une métaphorisation de la communication politique selon laquelle elle est conçue et comprise comme une guerre. Plus précisément, la politique et la communication politique sont ainsi considérées comme une forme figurée de bataille ou de guerre.

A ce propos, mais sur un plan plus général, Lakoff et Johnson ${ }^{1}$ soutiennent même que toutes les formes de discussion institutionnalisées dans nos sociétés modernes doivent être vues comme des applications de ce qu'ils appellent "la métaphore structurante". La discussion rationnelle, c'est la guerre. Voici en quels termes, ils proposent leur thèse:

(...) notre conception de la discussion mais aussi notre manière de la mener se fondent sur notre connaissance et sur notre expérience du combat physique. Même si vous n'avez jamais participé de votre vie à une bagarre, et encore moins à une guerre, vous avez participé à des discussions dès le moment où vous avez commencé à parler, et vous concevez et vous menez ces discussions en vous conformant à la métaphore. La discussion, c'est la guerre parce qu'elle fait partie intégrante du système conceptuel de la culture dans laquelle vous vivez ${ }^{2}$.

1 George Lakoff et Mark Johnson, Les métaphores dans la vie quotidienne, Paris, Editions de Minuit, 1985.

2 lbid., p. 72-73. 
Lakoff et Johnson vont jusqu'à suggérer que la distinction entre la conversation et la discussion réside dans l'intégration dans la seconde d'un élément d'affrontement:

La conversation devient une véritable discussion quand chacun des interlocuteurs consacre l'essentiel de son énergie conversationnelle à essayer de discréditer la position de l'autre tout en s'efforçant de conserver la sienne. La discussion demeure une conversation, mais la coopération polie, comme critère du maintien de la structure conversationnelle, peut être remise en cause si la discussion s'enflamme'.

La communication politique est manifestement l'un des principaux lieux où s'opère ce passage de la conversation à la discussion. C'est ce qui explique que la communication politique puisse être conçue et perçue comme une bataille, comme une guerre. "Comme", c'est-à-dire à la façon d'une guerre ou, si l'on veut, comme une guerre métaphorique.

Cette métaphorisation de la politique et la présence soutenue au sein du discours politique de métaphores guerrières ouvrent, sur un plan plus général, à une certaine caractérisation de la communication politique: celle qui détermine la politique comme une entreprise de communication et, encore plus spécifiquement, la communication politique comme un affrontement. Telle qu'ainsi abordée, la communication politique (political communication) est devenue, ces dernières années, tout autant en Amérique du Nord qu'en Europe, un secteur important de la recherche en communication "publique" ou "sociale". Dans la foulée de cette émergence, des chercheurs en viennent à proposer une définition de la politique essentiellement en terme de communication et une caractérisation de cette communication politique en terme de confrontation entre discours opposés. Voici, par exemple, la définition que donne Dominique Wolton de la communication politique:

(...) l'espace où s'échangent les discours contradictoires des trois acteurs qui ont la légitimité à s'exprimer publiquement sur la politique et qui sont les hommes politiques, les journalistes et l'opinion publique au travers des sondages ${ }^{2}$.

1 lbid., p. 89.

2 Dominique Wolton, "La communication politique: construction d'un modèle", in Hermes. Cognition, Communication, Politique, 4: Le Nouvel Espace Public, Paris, Éditions du CNRS, 1989, p. 27-42. (Une version anglaise de ce texte a également 
Cette conception de la politique et cette détermination plus précise de la communication politique dégage un champ plus spécifique de recherche dont l'exploration pourrait servir, je crois, à mieux comprendre l'usage de métaphores guerrières dans le discours politique: celui de l'argumentation stratégique. J'ai d'ailleurs commencé à définir l'argumentation stratégique ${ }^{1}$. Disons seulement ici qu'elle est constituée de cette classe d'arguments ayant trait spécifiquement à la nature oppositionnelle de la communication politique, à ce que Wolton caractérise comme un lieu d'affrontement de discours contradictoires. Plus précisément, l'argumentation stratégique dénote les procédés discursifs qui se rapportent formellement (et aussi essentiellement sinon uniquement) à la nature conflictuelle ou polémique de la communication politique. Donnons pour seul exemple l'argument ad hominem. Tel qu'il est le plus fréquemment utilisé en politique, il ne sert pas au débat ou à l'échange désintéressé d'idées mais à l'attaque pure et simple de l'adversaire. Dans les termes de Lakoff et Johnson, un argument ad hominem ne relève pas de la conversation mais de la discussion, c'est-à-dire de l'affrontement: il a pour fonction de discréditer l'opposant. On voit immédiatement que la métaphore guerrière appartient au champ de l'argumentation stratégique de la communication politique.

été publiée: "Political Communication: the Construction of a Model", in European Journal of Communication, 5 (1), 1989, p. 9-28.

1 Gilles GAUTHIER, “Autopsie d'un débat politique: L'«interpellation» ParizeauBourassa. Argumentation stratégique et communication politique", (à paraître). Ibid., "L'homme et l'argument ad hominem dans la communication politique", (à paraître). 